\title{
Thin Organic Layers for Photography and Electronic Devices
}

\author{
Tadaaki Tani, ${ }^{1}$ Kazuhiko Seki, ${ }^{2}$ Keitaro Yoshihara, ${ }^{3}$ and Junichi Hanna ${ }^{4}$ \\ ${ }^{1}$ Frontier Core-Tech. Labs., Fuji Photo Film Co., Kaisei-machi, Ashigarakami-gun, Kanagawa-ken 258-8577, Japan \\ ${ }^{2}$ Department of Chemistry, Graduate School of Materials Science \& Institute for Advanced Research, Nagoya University, \\ Chikusa-ku, Nagoya-shi 464-8602, Japan \\ ${ }^{3}$ School of Materials Science, Japan Advanced Institute of Science \& Technology, Tatsunokuchi, Ishikawa 923-1292, Japan \\ ${ }^{4}$ Imaging Science and Engineering Laboratory, Tokyo Institute of Technology, Nagatsuda-cho, Midori-ku, Yokohama 226-8503, Japan
}

Received 30 March 2006; Revised 31 May 2006; Accepted 20 June 2006

\begin{abstract}
The dye sensitization in photography is achieved by thin layers of $J$-aggregated dyes on silver halide grains. Studies by Yoshihara et al. with picosecond and femtosecond spectroscopies have indicated that the migration of excitons in the dye layers plays important roles in their charge separation to inject electrons into the grains. Studies by Seki et al. with ultraviolet photoelectron spectroscopy have revealed that the electronic structure of the interface between dye layers and a substrate is characterized by the fact that the vacuum level of the former is lowered with respect to that of the latter by the electric double layer formed at the interface, and that this characteristic structure is generally valid for the interfaces between organic layers and substrates. Hanna et al. have confirmed the formation of such an electronic double layer at the interfaces between a liquid crystalline organic semiconductor and electrodes by analyzing the current voltage characteristics.
\end{abstract}

Copyright (c) 2006 Tadaaki Tani et al. This is an open access article distributed under the Creative Commons Attribution License, which permits unrestricted use, distribution, and reproduction in any medium, provided the original work is properly cited.

\section{INRODUCTION}

In most silver halide photographic materials, a photosensitive element is a silver halide grain covered with a monomolecular layer of $J$-aggregated dye molecules acting as spectral sensitizers, which absorb incident photons and then inject excited electrons to the grain to bring about spectral sensitization of the photographic process [1]. Especially in color films, we use a very thin tabular silver halide grain with large surface to volume ratio in order for the grain to adsorb the largest possible amount of sensitizing dye molecules in order to enhance the capture of incident photons. Photons captured by $J$-aggregated sensitizing dyes on a grain create excitons, and created excitons migrate within the aggregates and then inject to the grain electrons available for the formation of a latent image center composed of a silver cluster. It is therefore considered that a $J$-aggregated sensitizing dye layer on a silver halide grain is an effective "light-harvesting" system and is regarded as an appropriate subject of this special issue.

Since $J$-aggregated sensitizing dye molecules on a silver halide grain are regarded as a thin organic layer, fruitful knowledge accumulated during various investigations on spectral sensitization in photography for many years is expected to be valuable for the research and development of electronic devices with thin organic layers such as organic field-effect transistors, organic light-emitting devices, solar cells with thin organic layers. Common phenomena taking place in these devices are activities of electrons, positive holes, and excitons in thin organic layers in addition to electronic charge transfer processes across the interfaces between thin organic layers and substrates.

This paper is initiated by an explanation of dye sensitization in photography and solar cells to point out two important factors for the photoelectric behavior of thin organic layers, that is, the behavior of excitons and the electronic structure of the interface between organic layers and substrates. Then, descriptions are made on recent knowledge of the behavior of excitons in thin layers of $J$-aggregated dyes in photography, and the electronic structure and charge transfer across the interfaces between organic layers and substrates.

\section{DYE SENSITIZATION IN PHOTOGRAPHY AND SOLAR CELL}

Figure 1 illustrates the electronic structures of the interfaces between organic layers and substrates for typical devices including dye sensitization in photography [1], a solar cell with a thin organic layer, and an organic light-emitting device (OLED). The replacement of silver halide by titanium 


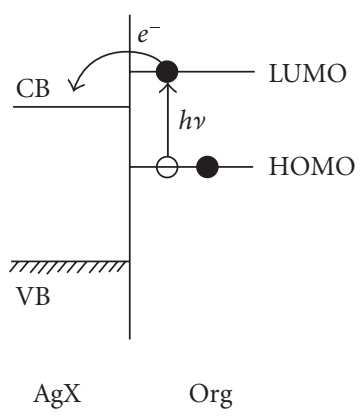

Photography

(a)

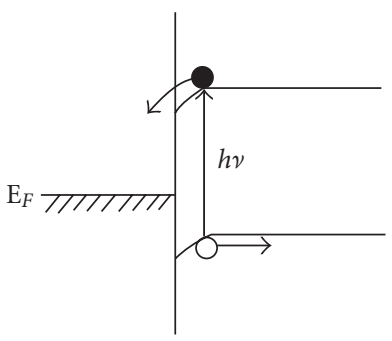

Metal

Solar cell

(b)

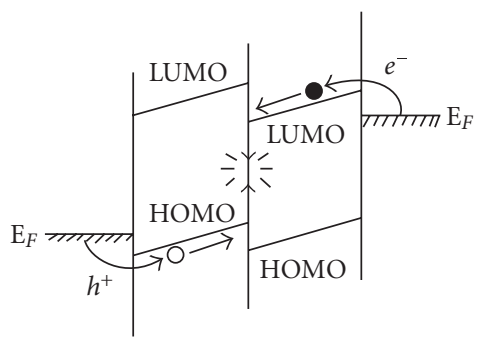

$\begin{array}{cccc}\text { Metal } & \text { Org } & \text { Org } & \text { Metal } \\ (\mathrm{HTL}) & (\mathrm{ETL})\end{array}$

OLED

(c)

FIGURE 1: Electronic structures of the interfaces between organic layers and substrates in photography, solar cell, and organic light-emitting device (OLED), where CB and VB are the conduction band and the valence band, respectively, LUMO and HOMO are the lowest unoccupied molecular orbital and the highest occupied one, respectively, $E_{F}$ is the Fermi level of a metal, AgX is silver halide, Org is organic layer, and HTL and ETL are hole transport and electron transport layers, respectively.

dioxide in the electronic structure for photography gives the electronic structure for a dye-sensitized solar cell as proposed by Hagfeldt and Grätzel [2] and Grätzel [3].

A color negative film is composed of photosensitive layers with $\sim 20 \mu \mathrm{m}$ thickness in total on a film base with $\sim 100 \mu \mathrm{m}$ thickness. The photosensitive layers contain three major layers, which are coated one on another and are sensitive to lights of three primary colors (i.e., blue, green, and red). Each major layer is composed of three sublayers with different sensitivity. Photosensitive elements in color films are silver halide grains. Silver halide grains in each major layer are covered with sensitizing dye layers having sensitivity to the light of one of the three primary colors. On exposure, the dye layers absorb a sufficient number of photons to create excitons, which migrate within the layers and are subjected to the charge separation whereby electrons are injected into the grains. In this way, a latent image center composed of a silver cluster is formed on the grain, and initiates photographic development, by which the grain is reduced to form a silver grain. In a color negative film, chemical reactions in its blue-, green-, and red-sensitive layers during the development form the dyes with colors of yellow, magenta, and cyan, respectively, each of which is complementary to the corresponding color of the absorbed light.

The phenomenon, which makes a silver halide grain sensitive to the light absorbed by dye layers on the grain, is called spectral sensitization or dye sensitization, and was discovered for silver halide photographic materials by Vogel [4]. Such layers may consist of monomeric molecules or dye aggregates. A dye in the state of a special kind of such aggregates, termed $J$-aggregate, gives a sharper absorption band at longer wavelength than that in monomeric state. $J$-aggregated cyanine dye molecules are adsorbed to the surface of silver halide with edge-on orientation to form a monomolecular dye layer with thickness of about $1 \mathrm{~nm}$ [1].

Silver halide grains used in modern color films are tabular and very thin, having very large surface-to-volume ratio and thus suitable to adsorb large amounts of $J$-aggregated

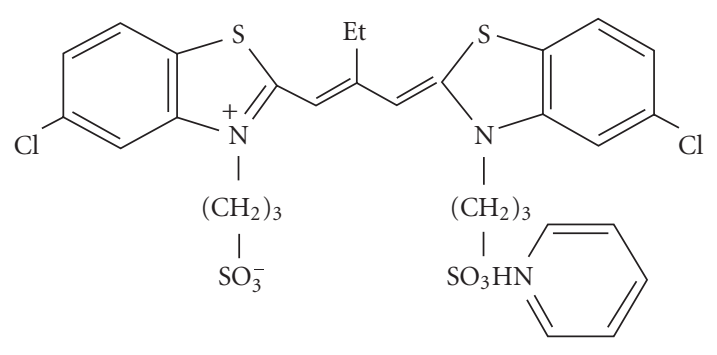

FIgURE 2: Chemical structure of a typical sensitizing dye used for photographic materials.

sensitizing dyes. When light-excited, a $J$-aggregate of dye molecules on a grain absorbs an incident photon to create an exciton, which migrates within the aggregate and is subjected to its charge separation to inject into the grain an electron available for the formation of a latent image center in collaboration with an interstitial silver ion.

A dye-sensitized solar cell as proposed by Hagfeldt and Grätzel [2] and Grätzel [3] and studied by many groups of workers is composed of a layer of sintered titanium dioxide grains covered with sensitizing dye molecules. In order to increase the amount of the dye in the layer, the grains are as small as $\sim 20 \mathrm{~nm}$ in diameter. Electrons are excited in dye molecules, injected into the grains, migrate among the grains, and reach an ITO anode. Resultant positive holes in the dye molecules are transferred to a cathode through a redox system in an electrolyte solution.

While sensitizing dyes used for photographic materials are mostly cyanine dyes (Figure 2) capable of forming $J$ aggregates on the surface of silver halide grains, a typical sensitizing dye used for a dye-sensitized solar cell is called N3 (Figure 3), being quite different from cyanine dyes in that an $\mathrm{N} 3$ molecule contains a ruthenium atom in it, and does not form any aggregate with other molecules on titanium dioxide grains $[2,3]$. 
In photography, an electron in the highest occupied molecular orbital (HOMO) in a dye molecule is excited to the lowest unoccupied molecular orbital (LUMO), and is transferred to the conduction band of silver halide as shown in Figure 1. The quantum yield of the electron transfer as a function of the energy gap was studied for many dyes on the basis of the Marcus theory [5]. The energy gap in this system is the height of LUMO of a sensitizing dye with respect to the bottom of the conduction band of silver halide, and is represented by its reduction potential under the assumption that the reduction potentials of dyes with similar structures in monomeric state in solutions are nearly parallel with their LUMO levels in condensed states on the grains. Figure 4 shows the energy gap dependence of the quantum yield of the electron transfer. As represented by open circles in this figure, the observed quantum yields are nearly unity for the dyes, whose energy gaps are larger than the threshold value. Among the dyes with the energy gaps smaller than the threshold value, the quantum yields decreased steeply with decreasing the energy gap. The comparison of the open circles with the solid line, which is derived from the Marcus theory with adjusted parameters, indicates that the electron transfer takes place in the absence of the inverted region with small rearrangement energy.

The above-stated results indicate that $J$-aggregated sensitizing dye in photography is one of the devices with thin organic layers, in which the charge separation is determined by the behaviors of excitons and the electronic structures of their interfaces with substrates. In the following part of this paper, descriptions are made on the behaviors of excitons in $J$-aggregated cyanine dyes on silver halide grains and the electronic structures of the interfaces between organic layers and substrates.

\section{BEHAVIOR OF EXCITONS IN THIN LAYERS OF $J$-AGGREGATED DYES IN PHOTOGRAPHY}

Although quantum yields of dye sensitization by many sensitizing dyes in photography are usually low owing to the fact that excited electrons in the dye molecules are below the bottom of the conduction band of silver halide, the quantum yields of many sensitizing dyes can be improved to be nearly unity by supersensitization as illustrated in Figure 5 $[1,5]$. Namely, a supersensitizer molecule injects an electron into a $J$-aggregate of sensitizing dyes in the excited state, and thus enhances the charge separation (i.e., the electron transfer from the excited $J$-aggregate to the conduction band of silver halide). It is noted that the ratio of the amount of a supersensitizer to that of a sensitizing dye for achieving effective supersensitization is usually very small owing to the fact that only one supersensitizer molecule per a $J$-aggregate composed of many sensitizing dye molecules is needed for the supersensitization, since an exciton in a $J$-aggregate can migrate and reach a supersensitizer molecule at the end of a J-aggregate as illustrated in Figure 6. This is one of the most important reasons for the fact that supersensitization is particularly effective for sensitizing dyes in $J$-aggregated state.

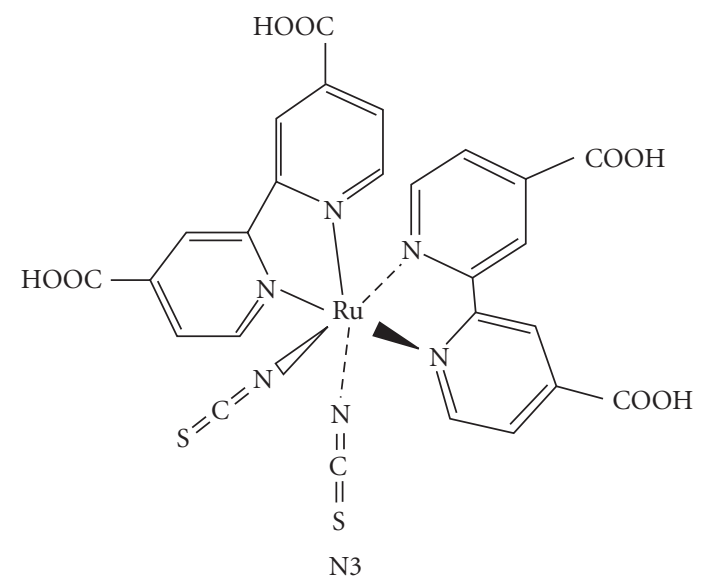

FIgure 3: Chemical structure of a typical sensitizing dye N3 used for dye-sensitized solar cells.

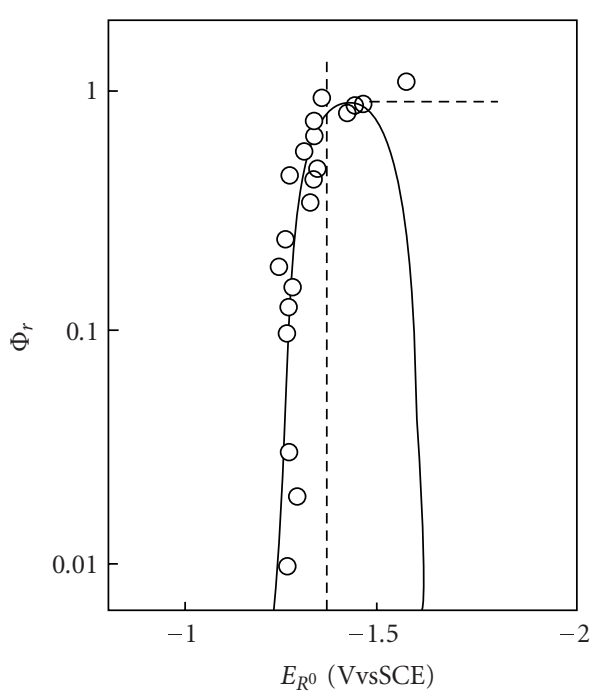

FIGURE 4: Quantum yield $\Phi_{r}$ of the electron transfer to silver bromide grains in dye sensitization as a function of its energy gap between the LUMO level of a dye and the bottom of the conduction band of silver bromide. The reduction potential of a dye $\left(E_{R^{0}}\right)$ on the abscissa is used to indicate the relative height of LUMO of the dye on the basis of the linear relationship between them [2]. The solid line is derived from the Marcus theory with rearrangement energy of $0.05 \mathrm{eV}$.

Picosecond spectroscopy was used to measure the fluorescence lifetime of sensitizing dyes on silver halide grains for the evaluation of the rate constants of the electron transfer and deactivation processes [5-9]. Figure 7 shows the result reported by some of the present authors [9]. As seen in this figure, the electron transfer from excited dye molecules in $J$-aggregated state to silver halide grains takes several tens picoseconds, and becomes to be slower as the size of $J$ aggregates increases. As illustrated in Figure 8, an exciton migrates and is trapped by the molecule at the end of a $J$ aggregate, which acts as a trap for an exciton and enhances its charge separation to bring about the electron injection into a 


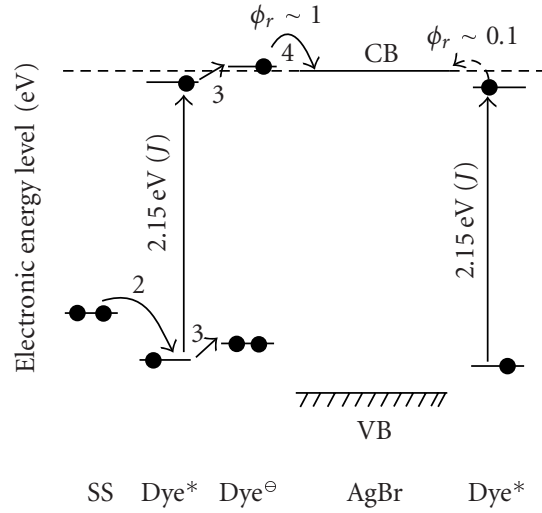

(a)<smiles>[X][N+]1(CC)CCCC1=Cc1ccc2ccccc2[n+]1CC</smiles><smiles>CN(C)c1ccc(C=Cc2nc3ccccc3s2)cc1</smiles>

SS

(b)

FIGURE 5: An illustration showing the mechanism of supersensitization for an inefficiently sensitizing dye (Dye) by a supersensitizer (SS) on silver bromide, where $\Phi_{r}$ is the quantum yield of the electron transfer in dye sensitization, and Dye* and Dye ${ }^{\ominus}$ are the dye in the excited state and the dye in one-electron-surplus state, respectively.
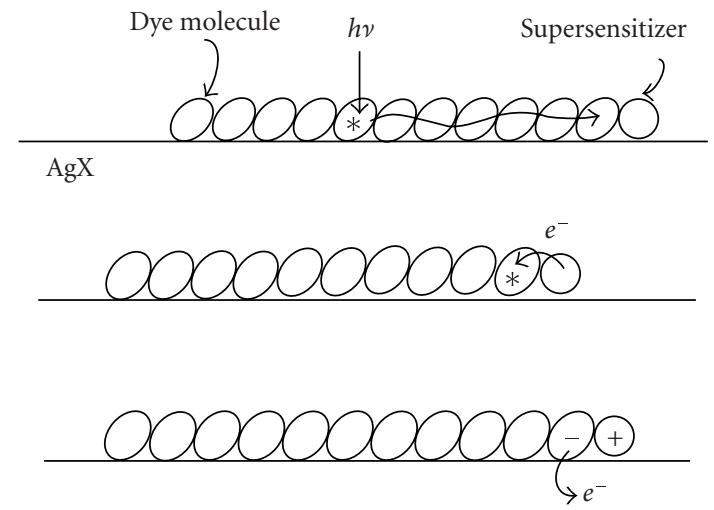

Figure 6: An illustration showing the role of an exciton in supersensitization taking place in a $J$-aggregate.

silver halide grain, supporting the idea proposed by West and Carroll 50 years ago $[10,11]$. Since the concentration of the molecules at the ends of $J$-aggregates decreases with increasing the size of $J$-aggregates, the rate of the electron injection decreases with increasing the size of $J$-aggregates.

The above-stated results characterize the behavior of excitons in thin organic layers. Namely, the absorption of a photon by an organic layer creates an exciton in it. The exciton migrates within the layer and dissociates when it can reaches a site, where the charge separation is possible. This knowledge about exciton migration in thin organic layers is supplied by the study on the exciton migration in thin layers of $J$-aggregated sensitizing dyes on silver bromide grains by Oh, Yoshihara et al. with femtosecond spectroscopy [12] .

When randomly oriented $J$-aggregates are exposed to a polarized light beam, each aggregate is excited with the probability, which depends on the angle between the electric field vector of the light beam and the transition moment of the aggregate. Then, a time-resolved measurement was made of the components of the fluorescence intensity of the aggregate

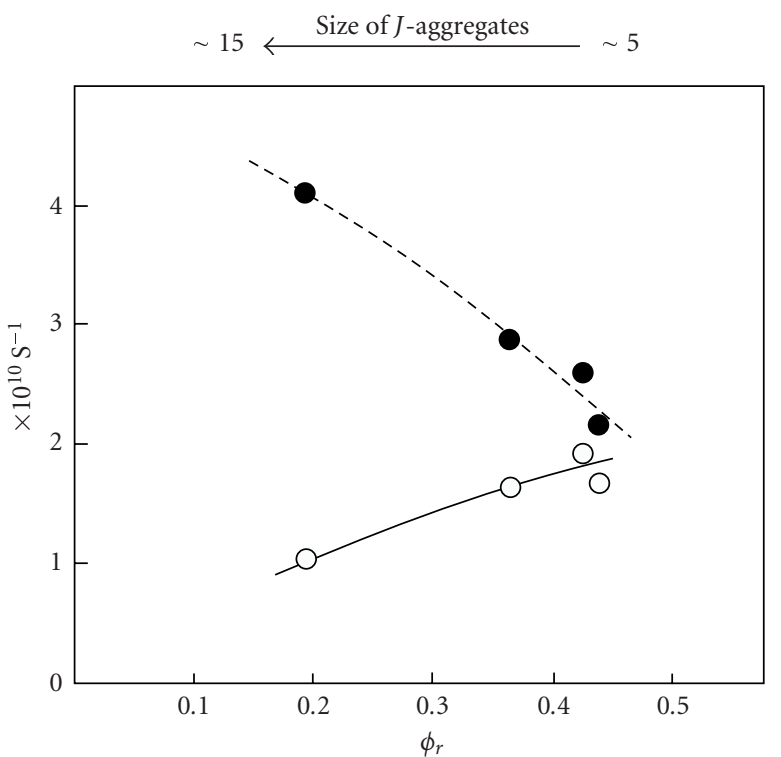

Figure 7: The rate constants of the electron transfer process $(\mathrm{ks} ; \bigcirc)$ and competing deactivation processes $(\mathrm{kL} ; \mathbf{O})$ in dye sensitization of silver bromide grains by the dye in Figure 2. As indicated on the abscissa, the quantum yield of dye sensitization $(\Phi r)$ decreases with increasing the size of the $J$-aggregates of the dye.

in Figure 2 with the transition moment parallel and perpendicular to the electric field vector of the exciting light beam ( $I_{\|}$and $I_{\perp}$, resp.). The polarization factor $r(t)$, as defined by $\left[I_{\|}(t)-I_{\perp}(t)\right] /\left[I_{\|}(t)+2 I_{\perp}(t)\right]$, is 0.4 at $t=0$, and decreases when excitons migrate among $J$-aggregates with different orientation. As shown in Figure 9, $r(t)$ decayed with two components, and the decay rate of fast component increased with increasing the surface coverage of the grains by the aggregates. Thus, the obtained result indicated that some of excitons created in $J$-aggregates of the dye migrated, not only within the aggregate, but also to other aggregates with 


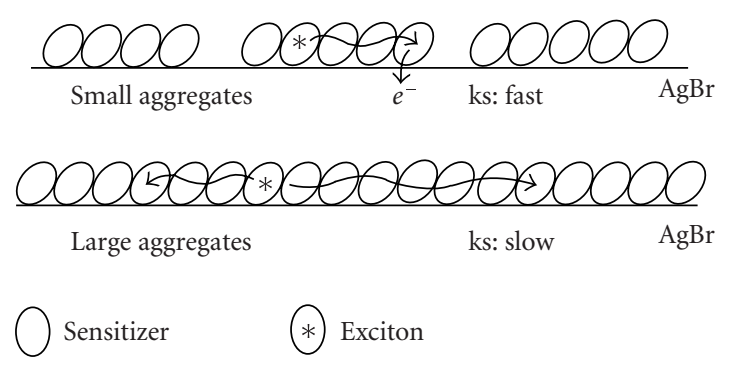

FIgURE 8: An illustration showing the role of an exciton in its charge separation (i.e., the electron transfer for dye sensitization) in a $J$ aggregate with variation of size, where $\mathrm{k}_{s}$ is the rate constant of the electron transfer.

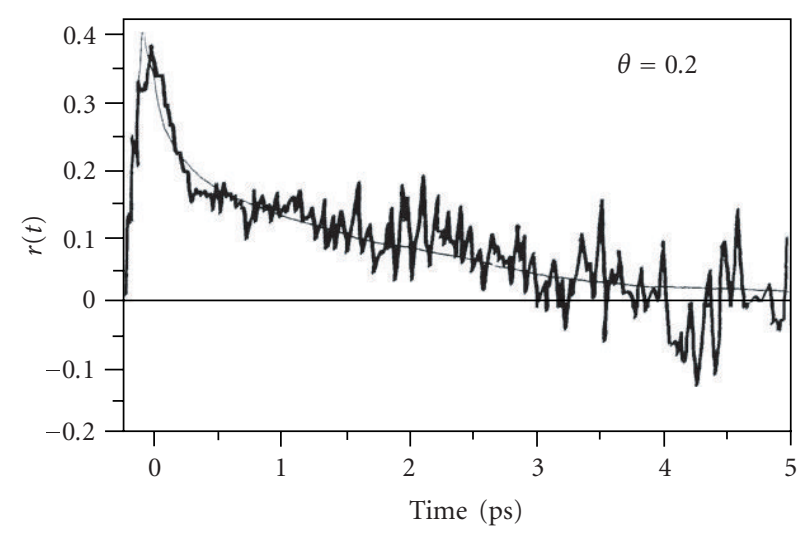

(a)

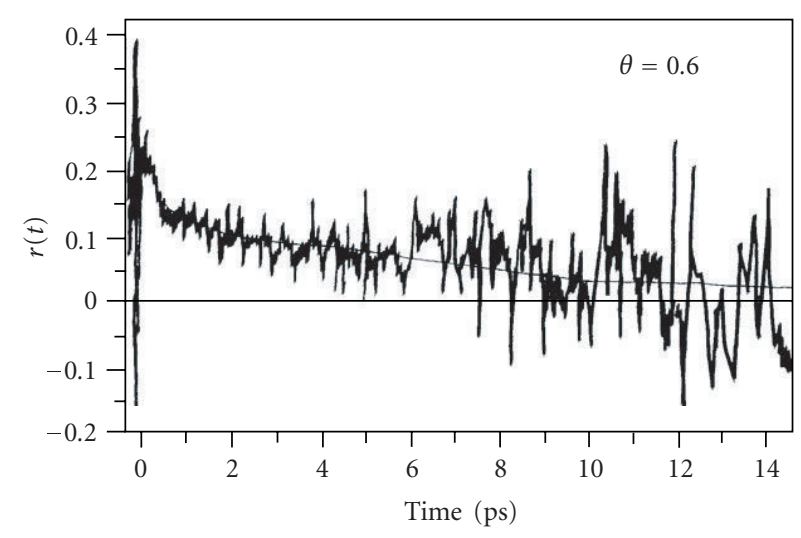

(b)

FIGURE 9: The polarization function $r(t)$ as derived from the timeresolved fluorescence intensity of the dye in Figure 2, which formed $J$-aggregates on silver bromide grains with variation of surface coverage $(\theta)$.

different orientation within one picosecond. Taking into account the fact that it takes several tens picoseconds for an exciton to dissociate to inject an electron into a silver halide grain after it migrates among aggregates, it is considered that an exciton could migrate to a long distance among $J$ aggregates of sensitizing dyes on silver bromide grains.

\section{ELECTRONIC STRUCTURE AND CHARGE TRANSFER ACROSS INTERFACE BETWEEN ORGANIC LAYER AND SUBSTRATE}

The electronic structures of materials have been evaluated by various methods including molecular orbital calculation, polarographic half-wave potential, phase-selective second harmonic voltammetry, and ultraviolet photoelectron spectroscopy (UPS) [13]. Among them, UPS is the only way which gives the electronic energy levels in a material with respect to its vacuum level. Seki, Tani, and collaborators use the UPS apparatus, where a sample in a vacuum chamber emits external photoelectrons to give a current in a circuit when it is exposed to light. The measurement of the current as a function of the retarding voltage makes it possible to evaluate the distribution of the kinetic energy (i.e., the energy distribution curve) of the external photoelectrons [13].

The electronic structure of several merocyanine dyes on a silver bromide layer has been studied [14]. In the first place, the energy distribution curve of the silver substrate was measured using its Fermi level as the standard. Then, silver bromide was evaporated on the substrate without breaking the vacuum, and the energy distribution curve of silver bromide was measured with respect to the Fermi level of the silver substrate. Finally, a merocyanine dye was evaporated on the silver bromide layer without breaking the vacuum, and the energy distribution curve of the dye was measured with respect to the Fermi level of the substrate.

Figure 10 shows the obtained electronic structure of the interfaces between silver bromide and merocyanine dyes with respect to the Fermi level of the silver substrate. Since it was striking that the vacuum levels of all the dyes studied were situated to be considerably below the vacuum level of silver bromide, it was important to compare this result with the photographic behaviors of the dyes studied. According to the UPS measurements, Dyes A and B should be sensitizing dyes, since their LUMO levels are above the bottom of the conduction band of silver bromide, and Dyes C and D should be desensitizing dyes, since their LUMO levels are below the bottom of the conduction band of silver bromide. In good accord with this prediction, it was confirmed through photographic tests that Dyes A and B were good spectral sensitizers, while Dyes $C$ and D were desensitizers [1]. This result directly proved the electron transfer mechanism of dye sensitization $[1,15]$ for the first time, and also proved the result that the vacuum level of a dye does not coincide with that of its substrate, and that the vacuum levels of the dyes are situated to be below the vacuum level of the substrate (i.e., silver bromide).

Seki and coworkers have considered that this result is important for the design of devices with organic layers, and extensively studied if this result is universally valid for the interface between an organic layer and an electrode by studying many organic compounds with widely varied ionization energy, although they have not included dyes in monomeric or $J$-aggregated state in their studies. It was found that the vacuum level of an organic layer is lowered with respect to that of an electrode for almost all the combinations between 


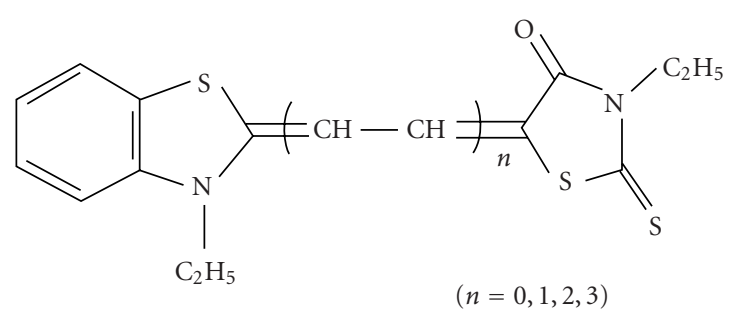

(a)

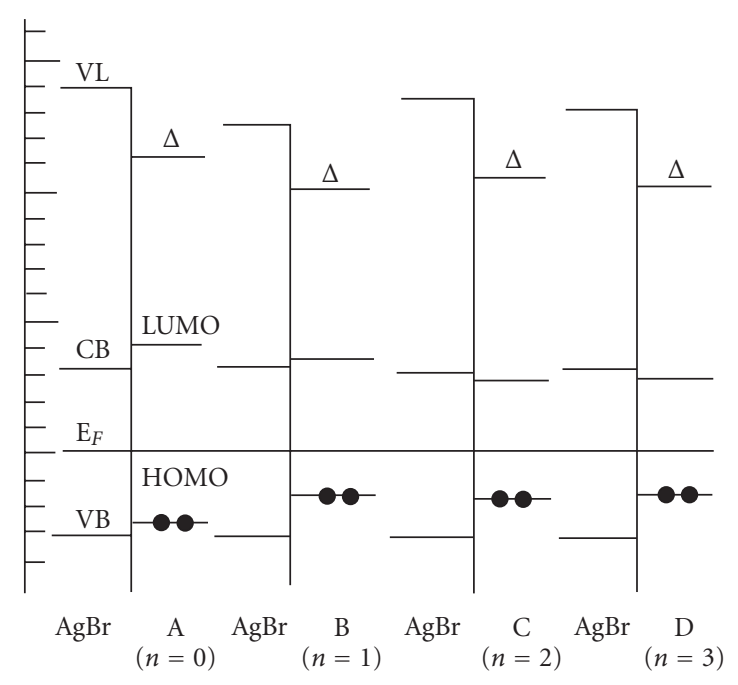

(b)

FIGURE 10: The electronic structure of the interfaces between merocyanine dyes having different chain lengths and silver bromide as determined by UPS measurement, where VL, $\mathrm{E}_{F}, \mathrm{CB}$, and VB are the vacuum level, Fermi level, conduction band, and valence band, respectively, LUMO and HOMO are the lowest unoccupied and the highest occupied molecular orbitals, respectively, and $\Delta$ is the displacement of the vacuum level of a dye with respect of the vacuum level of silver bromide.

organic layers and electrodes when they are in contact with each other [16-19]. This result indicates that the contact between an electrode and an organic layer forms the electric double layer at the interface, which lowers the electronic energy levels in an organic layer with respect to those in an electrode.

Several origins, which should cause the formation of such an electric double layer at the interface, have been discussed [16-19]. Among them, one of the most probable origins might be the pushback effect. Usually, the spillover of electrons out of the surface of a metal forms an electric double layer having negative and positive charges at the outside and inside of the surface. Then, the contact of an organic layer with a metal electrode depresses the spillover of electrons, substantially bringing about the formation of the electric double layer with a dipole, which lowers the electronic energy levels in an organic layer with respect to those in an electrode.

The result is of basic importance in the development of various devices with organic layers which should have high carrier mobility, large area, and low cost. The amorphous organic semiconductors hitherto known afford layers having large area and low cost but only low carrier mobility, since it is difficult for carriers to move smoothly among randomly oriented molecules in amorphous materials. On the other hand, crystalline organic semiconductors afford layers having high carrier mobility but small area and high cost. In order to fulfill all three requirements, Hanna and Funahashi have developed liquid crystalline organic semiconductors [20-23]. For this study, 2-(4'octylphenyl)-6-dodecyloxynaphthalene (8-PNP-O12) have been used, which forms a smectic liquid crystal with high carrier mobility. This compound is especially suitable for studying the temperature-dependent current-voltage characteristics of the interface between an electrode and an organic layer, since the mobility of positive holes is as high as $2 \times 10^{-4}$ and $1.5 \times 10^{-3} \mathrm{~cm} 2 / \mathrm{Vs}$ in its smectic $\mathrm{A}$ and $\mathrm{B}$ phases and nearly independent of temperature and electric field.

The current in a semiconductor under the electric field as applied by an electrode usually exhibits several components including ohmic, injection-limited, and diffusionlimited ones with increasing the applied voltage [24-26]. There are two models for the injection of carriers from an electrode to a semiconductor, that is, the Schottky injection with thermal activation of carriers and the tunnel injection. It is therefore expected that the temperature dependence of the injection-limited current according to the Schottky model gives the barrier height for the charge injection.

Investigations of the interfaces of electrodes of platinum, gold, and ITO with 8-PNP-O12 layers in liquid crystalline state confirmed that the observed currents at a certain electric field region were due to the injection of positive holes from the former to the latter according to the Schottky model [27]. The plots of the currents due to the Schottky injection exhibited straight lines. Slopes of which gave height of the energy barriers for the injection of positive holes from electrodes to 8-PNP-O12. According to the Schottky model, the barrier height decreased linearly with the square root of the electric field. Thus, the extrapolation of the straight line to the ordinate gives the barrier height for the positive hole injection, that is, the energy gap between the Fermi level of an electrode and the HOMO level of the compound in the absence of the applied electric field.

The observed barrier heights were much larger than the corresponding energy gaps, indicating the formation of the electric double layer at the interface between 8-PNP-O12 and electrodes [28]. This result provides direct evidence that the vacuum level of an organic layer is lowered with respect to that of an electrode when they are in contact with each other. This result also indicates that the electric double layer formed at the interface makes it difficult to inject positive holes from an electrode into an organic layer as illustrated in Figure 11.

They have tried to enhance the positive hole injection by diminishing the electric double layer with the adsorption of a 


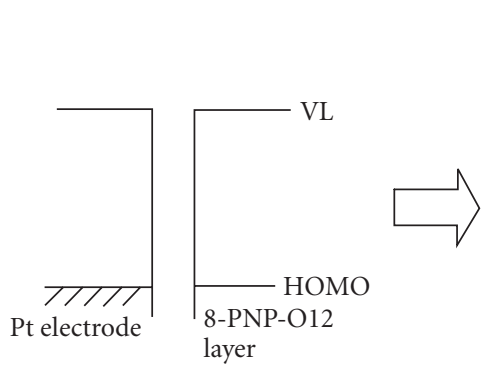

(a)

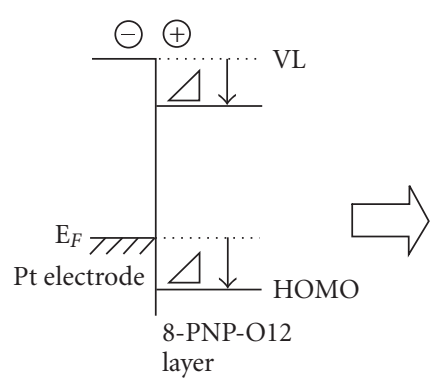

(b)

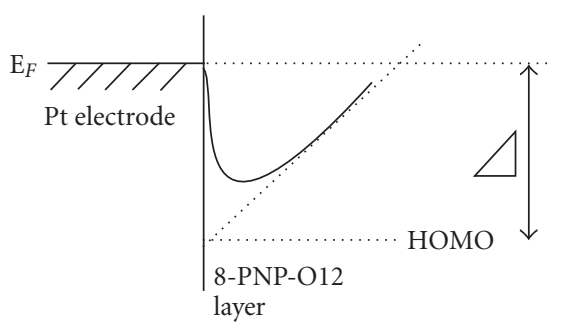

(c)

FIGURE 11: Electronic structure of the interface between a platinum electrode and 8-PNP-O12 layer before and after they come in contact with each other in the absence of applied voltage ((a) and (b), resp.) and in the presence of applied voltage (c), where VL is the vacuum level.

compound having an electric dipole with opposite direction such as a nitrothiophenol and observed that the adsorption of a thiophenol to Au electrode could increase the current due to the injection of positive holes into the electrode [29].

\section{REFERENCES}

[1] T. Tani, Photographic Sensitivity; Theory and Mechanisms, Oxford University Press, New York, NY, USA, 1995.

[2] A. Hagfeldt and M. Grätzel, "Light-induced redox reactions in nanocrystalline systems," Chemical Reviews, vol. 95, no. 1, pp. 49-68, 1995.

[3] M. Grätzel, "Photoelectrochemical cells," Nature, vol. 414, no. 6861, pp. 338-344, 2001.

[4] H. W. Vogel, "Uber die lightempfindlichkeit der Bromsilbers for die sogenannten chemisch unwirksamen Farben," Chemische Berichte, vol. 6, p. 1302, 1873.

[5] T. Tani, T. Suzumoto, and K. Ohzeki, "Energy gap dependence of efficiency of photoinduced electron transfer from cyanine dyes to silver bromide microcrystals in spectral sensitization," The Journal of Physical Chemistry, vol. 94, no. 4, pp. 12981301, 1990.

[6] S. Daehne, F. Fink, E. Klose, K. Treuchner, G. Bach, and J. V. Grossmann, "Estimation of the rate constant for spectral sensitization of silver halides by J-aggregates," J. Signal AM., vol. 6, p. 105, 1978.

[7] D. V. Brumbaugh, A. A. Muenter, W. Knox, and G. Mourou, "Singlet exciton annihilation in the picosecond fluorescence decay of 1, 1' -diethyl-2,2' -cyanine chloride dye J-aggregate," Journal of Luminescence, vol. 31-32, part 2, pp. 783-785, 1984.

[8] K. Takahashi, K. Obi, I. Tanaka, and T. Tani, "Picosecond kinetics of spectral sensitization by a J-aggregated dye on $\mathrm{AgBr}$ microcrystals," Chemical Physics Letters, vol. 154, no. 3, pp. 223-227, 1989.

[9] T. Tani, T. Suzumoto, K. Kemnitz, and K. Yoshihara, "Picosecond kinetics of light-induced electron transfer from Jaggregated cyanine dyes to silver bromide microcrystals: effect of aggregate size," The Journal of Physical Chemistry, vol. 96, no. 7, pp. 2778-2783, 1992.

[10] W. West and B. H. Carroll, "Photo-conductivity in photographic systems I. Dye-sensitization of photo-conductivity," The Journal of Chemical Physics, vol. 15, no. 8, pp. 529-543, 1947.
[11] W. West and B. H. Carroll, "Energy transfer in the photosensitization of silver halide photographic emulsions: optical sensitization, supersensitization, and antisensitization," The Journal of Chemical Physics, vol. 19, no. 4, pp. 417-427, 1951.

[12] J.-W. Oh, S. Kumazaki, I. V. Rubtsov, T. Suzumoto, T. Tani, and K. Yoshihara, "Ultrafast energy transfer in J-aggregate on AgBr microcrystals: its dependence on dye coverage," Chemical Physics Letters, vol. 352, no. 5-6, pp. 357-362, 2002.

[13] T. Tani, K. Ohzeki, and K. Seki, "Phase selective second harmonic AC voltammetry and ultraviolet photoelectron spectroscopy for evaluation of electronic structures of cyanine dyes," Journal of the Electrochemical Society, vol. 138, no. 5, pp. 1411-1415, 1991.

[14] K. Seki, H. Yanagi, Y. Kobayashi, T. Ohta, and T. Tani, "UV photoemission study of dye/AgBr interfaces in relation to spectral sensitization," Physical Review B, vol. 49, no. 4, pp. 2760-2767, 1994.

[15] R. W. Gurney and N. F. Mott, "The theory of the photolysis of silver bromide and the photographic latent image," Proceedings of the Royal Society of London. Series A, vol. 164, no. 917, pp. 151-167, 1938.

[16] K. Seki, T. Tani, and H. Ishii, "Electronic structures of organicinorganic interfaces studied by UV photoemission," Thin Solid Films, vol. 273, no. 1-2, pp. 20-26, 1996.

[17] H. Ishii and K. Seki, "Energy level alignment at organic/metal interfaces studied by UV photoemission: breakdown of traditional assumption of a common vacuum level at the interface," IEEE Transactions on Electron Devices, vol. 44, no. 8, pp. 12951301, 1997.

[18] H. Ishii, K. Sugiyama, D. Yoshimura, E. Ito, Y. Ouchi, and K. Seki, "Energy-level alignment at model interfaces of organic electroluminescent devices studied by uv photoemission: trend in the deviation from the traditional way of estimating the interfacial electronic structures," IEEE Journal on Selected Topics in Quantum Electronics, vol. 4, no. 1, pp. 24-32, 1998.

[19] H. Ishii, K. Sugiyama, E. Ito, and K. Seki, "Energy level alignment and interfacial electronic structures at organic/metal and organic/organic interfaces," Advanced Materials, vol. 11, no. 8, pp. 605-625, 1999.

[20] M. Funahashi and J. Hanna, "Photoconductive behavior in smectic a phase of 2-(4' - heptyloxyphenyl)-6-dodecylthiobenzothiazole," Japanese Journal of Applied Physics, Part 2: Letters, vol. 35, no. 6 A, pp. L703-L705, 1996. 
[21] M. Funahashi and J. Hanna, "Fast ambipolar carrier transport in smectic phases of phenylnaphthalene liquid crystal," Applied Physics Letters, vol. 71, no. 5, pp. 602-604, 1997.

[22] M. Funahashi and J. Hanna, "Fast hole transport in a new calamitic liquid crystal of 2-(4' -heptyloxyphenyl)-6-dodecylthiobenzothiazole," Physical Review Letters, vol. 78, no. 11, pp. 2184-2187, 1997.

[23] M. Funahashi and J. Hanna, "First electronic conduction with high hole mobility in smectic a phase of a calamitic liquid crystal," Molecular Crystals and Liquid Crystals, vol. 304, pp. 429434, 1997.

[24] S. M. Sze, Physics of Semiconductor Devices, John Wiley \& Sons, New York, NY, USA, 2nd edition, 1981.

[25] P. E. Burrows, Z. Shen, V. Bulovic, et al., "Relationship between electroluminescence and current transport in organic heterojunction light-emitting devices," Journal of Applied Physics, vol. 79, no. 10, pp. 7991-8006, 1996.

[26] M. A. Baldo and S. R. Forrest, "Interface-limited injection in amorphous organic semiconductors," Physical Review B Condensed Matter and Materials Physics, vol. 64, no. 8, Article ID 085201, 17 pages, 2001.

[27] T. Toda, T. Takeuchi, J. Hanna, and T. Tani, "Electrical properties and electronics structure of the interface between electrode and liquid-crystalline semiconductor," Journal of the Society of Photographic Science and Technology of Japan, vol. 68, p. 390, 2005.

[28] T. Toda, J. Hanna, and T. Tani, to appear in Journal of Applied Physics.

[29] T. Toda, J. Hanna, and T. Tani, "A study on positive hole injection from electrode to liquid-crystalline semiconductors," in NIP22; 22nd International conference on digital printing tecnologies, pp. 7-22, Denver, Colorado, USA, September 2006. 


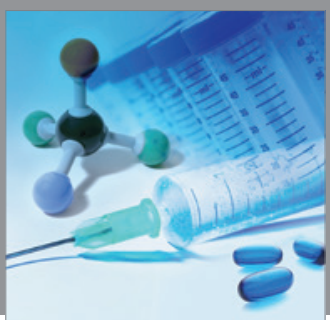

International Journal of

Medicinal Chemistry

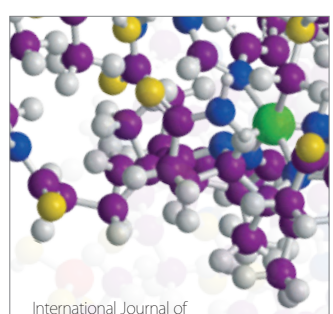

Carbohydrate Chemistry

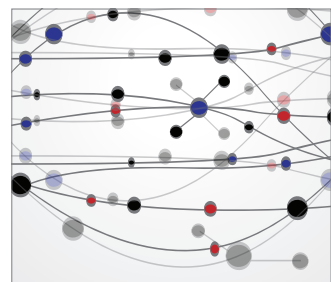

The Scientific World Journal
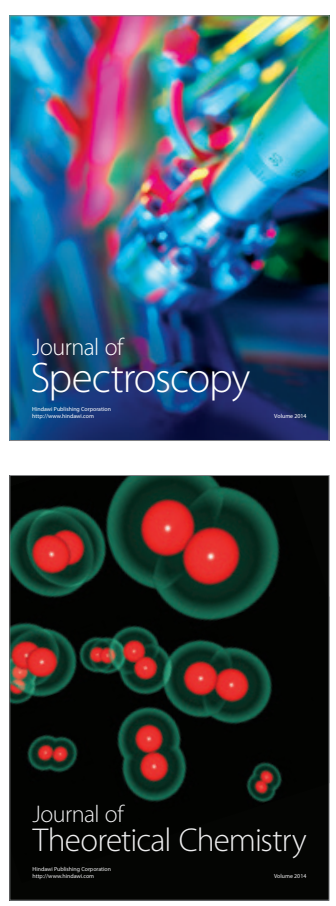
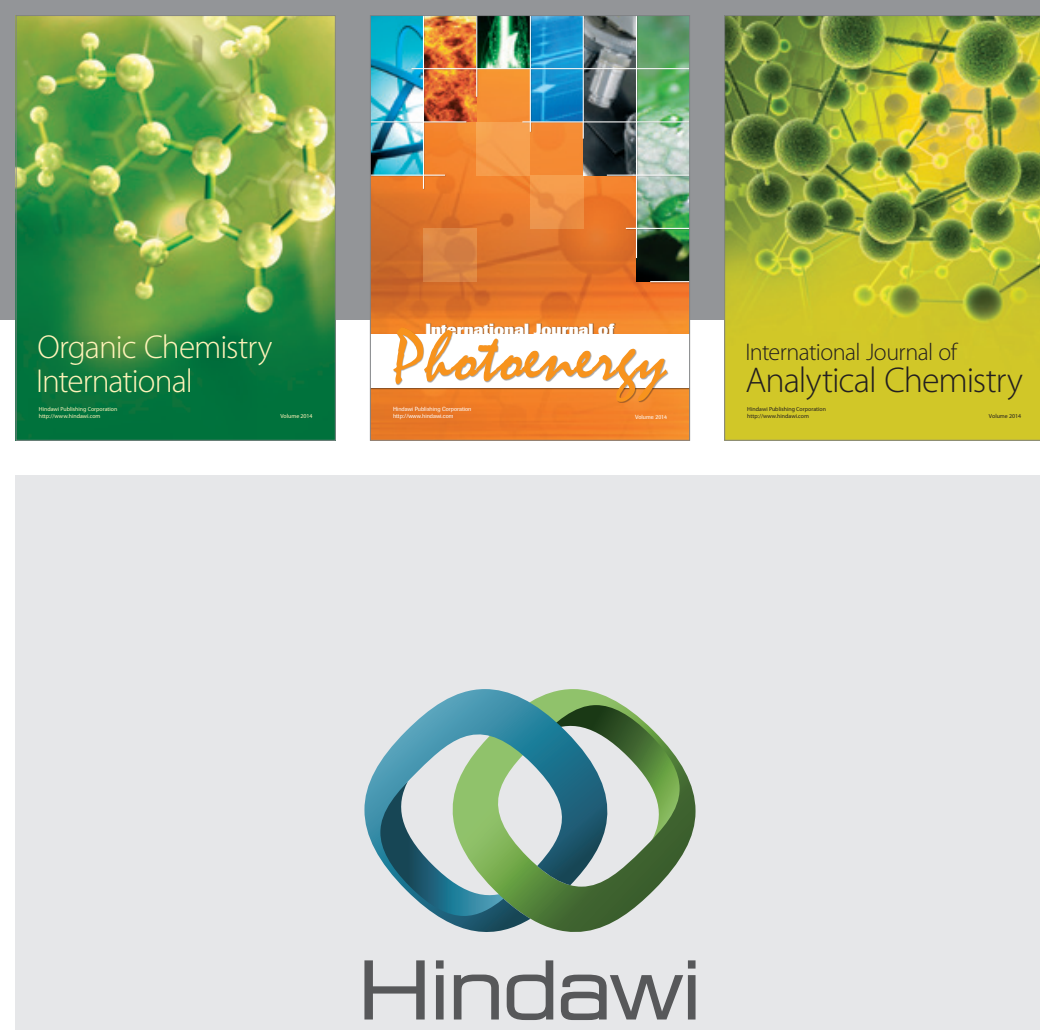

Submit your manuscripts at

http://www.hindawi.com
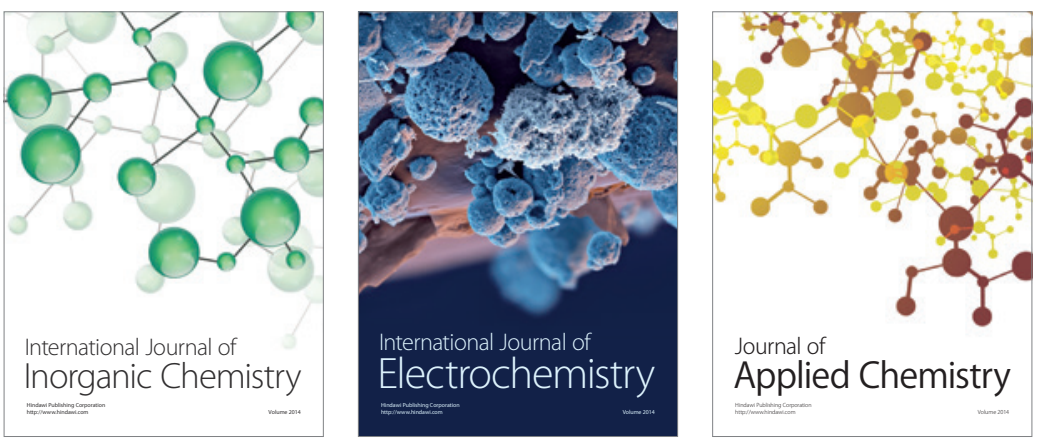

Journal of

Applied Chemistry
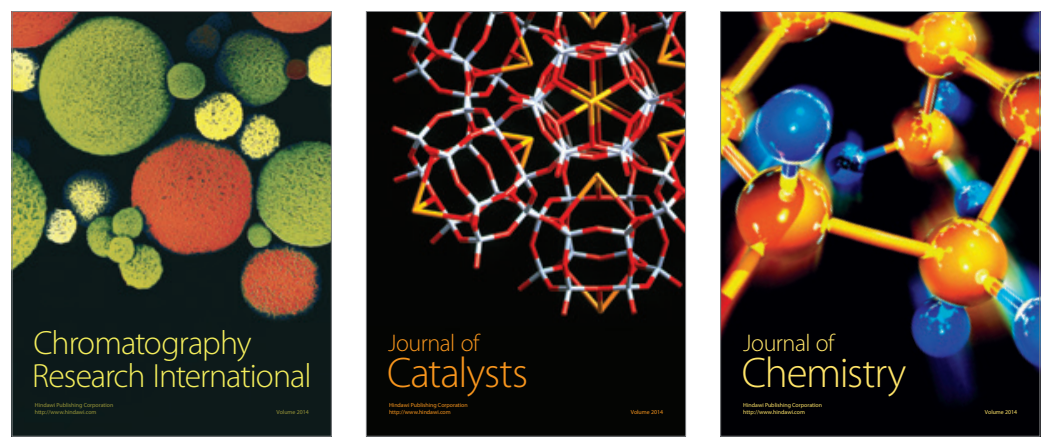
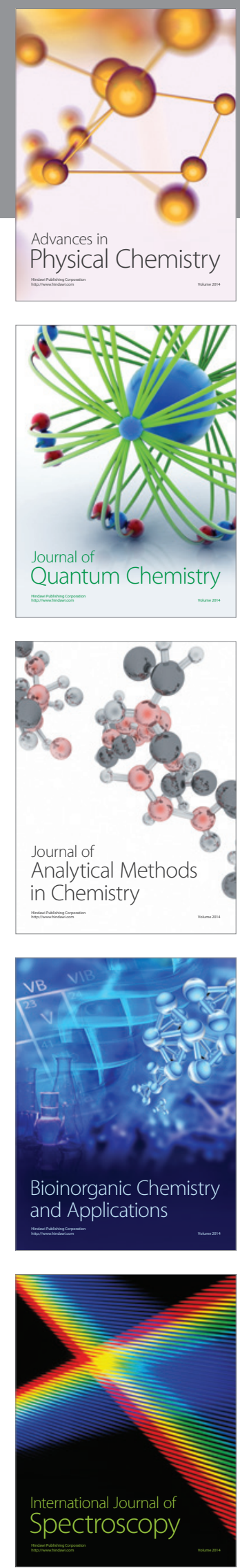